\title{
Study on Parameters Setting of Chan and Vese Model to Iris Segmentation
}

\author{
Ying Chen ${ }^{1, a^{*}}$, Fengyu Yang ${ }^{1, b}$ \\ ${ }^{1}$ School of Software, Nanchang Hangkong University, Nanchang, 330063, China \\ ac_y2008@163.com, brueyang@163.com
}

Keywords: Level set; Iris segmentation; Chan-Vese model; Parameter setting

Abstract. Iris recognition plays an important role in personal identification. The first and critical step in the process of an iris recognition system is iris segmentation. In this study, several aspects are done. Firstly, the fundamental of Chan-Vese level set is described. Secondly, the process of related parameters setting of CV model is analyzed when CV model is used to pupil and iris segmentation. Finally, some conclusions are drawn. Some iris image from Institute of Automation, Chinese Academy of Science (CASIA) - V1 and CASIA-V3 Interval are taken as experimental samples. The experimental results show that appropriate parameters setting will help improve iris image segmentation effect.

\section{Introduction}

Today, biometric recognition is a common and reliable way to authenticate the identity of a living person based on physiological or behavior characteristics. Iris recognition is one of the most reliable biometrics that uses iris characteristics of human eyes and plays an important role in accurate identification of individuals. Generally speaking, Iris recognition system mainly includes iris image preprocessing, iris feature extraction and iris feature classification.

Iris image preprocessing includes image evaluation, iris localization, normalization, and enhancement. Generally speaking, the first and, perhaps, the most important step of the processing procedure in an iris recognition system is iris segmentation or localization[1]. A variety of techniques have been developed for iris localization. Daugman[2] used integrodifferential operator to find the circular boundaries of an iris; Tan et al.[3] performed a clustering based coarse iris localization scheme to extract a rough position of the iris, then used a novel integro-differential constellation to constructed the localization of papillary and limbic boundaries; Puhan et al. [4] proposed a segmentation method based on the Fourier spectral density, which performed row-wise adaptive threshold results in iris region approximately, and further compute the circle parameters for the limbic and pupil boundaries in deterministic time.

However, in practical environment, iris boundaries are often not circular or elliptical because of non-ideal image acquisition and physiological factors in practical situation. Moreover, eyelids, eyelashes, specular reflections, defocused, deformed, off-angle, motion blur, low contrast, over saturation, partial images and gaze deviation will degrade the segmentation performance. In recent years, some other state-of-the-art iris segmentation methods based on active contour model has become the mainstream methods [5,6,7]. In 2001, Chan and Vese[8] proposed a CV model, which is an approximation for the Mumford-Shah[9] segmentation model by following the level set based curve evolution formulation. CV model can detect objects whose boundaries are not necessary defined by gradient, and it works on blurred and noisy images, with various shapes, without smooth pre-operation. In this paper, we will mainly focus on analyze influence of parameter setting to iris segmentation accuracy. 


\section{Description of Chan and Vese Model}

Assume that the evolving curve $C$ in $\Omega$, as the boundary of an open subset $\omega$ of $\Omega$, inside $(\mathrm{C})$ denotes the region $\omega$, and outside $(\mathrm{C})$ denotes the region $\Omega \backslash \varpi, u_{0}$ is the original image, and which is formed by two regions of approximatively piecewise-constant intensities. The energy function of $\mathrm{CV}$ model is

$$
\begin{aligned}
F\left(c_{1}, c_{2}, C\right)= & \mu \cdot \text { Length }(C) \\
& +\lambda_{1} \cdot \int_{\text {inside }(C)}\left|u_{0}(x, y)-c_{1}\right|^{2} d x d y \\
& +\lambda_{2} \cdot \int_{\text {ouside }(C)}\left|u_{0}(x, y)-c_{2}\right|^{2} d x d y
\end{aligned}
$$

where $\mu \geq 0, v \leq 0, \lambda_{1}, \lambda_{2}>0$ are fixed parameters. $c_{1}$ and $c_{2}$ are constants and they depending on $C, c_{1}$ is the average of $\mu_{0}$ inside $C$ and $c_{2}$ is the average of $\mu_{0}$ outside $C$, the length parameter $\mu$ is a scaling role since it determines the relative importance of the length term.

For the level set formulation of CV model, the curve $C$ is represented implicitly via a Lipschitz function $\phi$. Using the Heaviside function $H$, and the one-dimensional Dirac measure $\delta_{0}$, and defined, respectively, by

$$
H(z)=\left\{\begin{array}{ll}
1, & \text { if } z \geq 0 \\
0, & \text { if } z<0
\end{array}, \quad \delta_{0}(z)=\frac{d}{d z} H(z)\right.
$$

The energy function can be re-written in the following way.

$$
\begin{aligned}
F\left(c_{1}, c_{2}, \phi\right)= & \mu \int_{\Omega} \delta(\phi(x, y)) \mid \nabla \phi(x, y) d x d y \\
& +\lambda_{1} \cdot \int_{\Omega}\left|u_{0}(x, y)-c_{1}\right|^{2} H(\phi(x, y)) d x d y \\
& +\lambda_{2} \cdot \int_{\Omega}\left|u_{0}(x, y)-c_{2}\right|^{2}(1-H(\phi(x, y))) d x d y
\end{aligned}
$$

In order to compute the associated Euler-Lagrange equation for the unknown function $\phi$, the function $H$ and $\delta_{0}$ were slightly regularized, and denoted by $\mathrm{H} \varepsilon$ and $\delta \varepsilon$, respectively, as $\varepsilon \rightarrow 0$. The associated regularized function was denoted by $F_{\varepsilon}$ and defined by

$$
\begin{aligned}
F_{\varepsilon}\left(c_{1}, c_{2}, \phi\right)= & \mu \int_{\Omega} \delta_{\varepsilon}(\phi(x, y))|\nabla \phi(x, y)| d x d y \\
& +\lambda_{1} \cdot \int_{\Omega}\left|u_{0}(x, y)-c_{1}\right|^{2} H_{\varepsilon}(\phi(x, y)) d x d y \\
& +\lambda_{2} \cdot \int_{\Omega}\left|u_{0}(x, y)-c_{2}\right|^{2}\left(1-H_{\varepsilon}(\phi(x, y))\right) d x d y
\end{aligned}
$$

Keeping $c_{1}$ and $c_{2}$ fixed, and minimizing $\mathrm{F} \varepsilon$ with the respect of $\phi$, and which was deduced via the associated Euler-Lagrange equation. Parameterizing the descent direction by time $\mu \geq 0$, the equation $\phi(x, y, t)$ is derived as

$$
\begin{aligned}
& \frac{\partial \phi}{\partial t}=\partial_{\varepsilon}(\phi)\left[\mu \cdot \operatorname{div}\left(\frac{\nabla \phi}{|\nabla \phi|}\right)-\lambda_{1}\left(u_{0}-c_{1}\right)^{2}+\lambda_{2}\left(u_{0}-c_{2}\right)^{2}\right] \\
& \phi(x, y, 0)=\phi_{0}(x, y) \text { in } \Omega \\
& \frac{\partial_{\varepsilon}(\phi)}{|\nabla \phi|} \frac{\partial \phi}{\partial \vec{n}}=0 \text { on } \partial \Omega
\end{aligned}
$$

Where $\partial \vec{n}$ denotes the exterior normal to the boundary $\partial \Omega$, and $\partial \phi / \partial \vec{n}$ denotes the normal derivation of $\phi$ at the boundary.

\section{Related Parameters}

Keep $\lambda_{1}=\lambda_{2}=1, \varepsilon=1$, therefore, according to Eq. 4, the parameter of $\mu$ will directly influence segmentation result. However, in practical application, we should think about how to stop curve evolution. Aimed at mentioned above circumstances, we analyze parameters which may influence CV model, these parameters mainly contain three parameters.

(1) The length parameter $\mu$. It shows that the higher of smoothing term, the smoother of contour. Chan and Vese pointed that $\mu$ is a scaling role, if we have to detect all or as many objects as possible and of any size, then $\mu$ should be small, if we have to detect only larger objects, then $\mu$ should has to be larger. 
(2) Convergence threshold $(C T)$. Number of pixel used in the convergence test, a convergence test is performed at the end of each iteration. The bigger convergence threshold is indicates the weaker convergence condition and the more easily convergence the split curve is. In our following experiments, if during five consecutive iterations, the contour has not evolved more than the threshold value, the covergence is reached.

(3) Maximum number of iterations (MNOT): Maximum number of iterations after the algorithm should stop (if it has not converged already), the actual number of iterations is less or equal to MNOT.

The first parameter directly related with evolution function of $\mathrm{CV}$ model, the latter two parameters denote the termination requirement of control the evolution function of $\mathrm{CV}$ model. Hence, we will mainly focus on discuss the first parameter.

\section{Experimental results and discussions Analysis of segmentation performance}

The CV model is implemented using C++ (with OpenCV) and MATLAB platform, and CREASEG is utilized, which has originally developed by Dietenbeck et al. [10]. We make secondary development to this platform according to our practical need. Moreover, some iris images from CASIA-V1 and CASIA-V3 databases [11] are applied in experiments.

\section{Pupil Segmentation}

CV model can segment pupil area accurately in full accordance with the features of pupil itself, and do not need to assume that the shape of pupil is circle or ellipse (we can see that the shape of pupil in Fig. 1(c) is neither circle nor ellipse), besides, the speckles in pupil region have not influence CV model's fine location results.

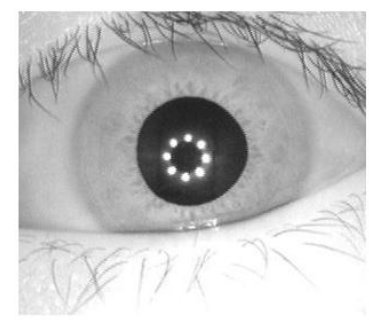

(a)

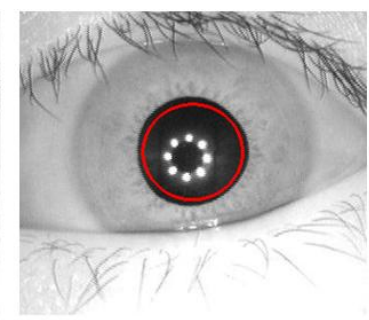

(b)

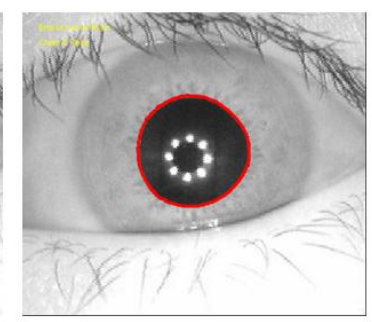

(c)

Figure 1. The process of fine pupil segmentaion. (a) Original iris image from CASIA-V3 Interval database, (b) Initial countour, (c) Final countour after 200 iterations (result of fine localization of pupil boundary). $\lambda_{1}=\lambda_{2}=1$, $\varepsilon=1, \mu=0.2 \cdot 255^{2}$.

However, excessive interference of eyelashes will influence location accuracy. As shown in Fig. 2(a), eyelashes extends to the pupil area, $\mathrm{CV}$ model obtains an accurate result after 20 times iterations, but $\mathrm{CV}$ model treat eyelashes as pupil incorrectly after 65 times iterations. The reason is that extended eyelashes and real pupil form a connected region. Consequently, the evolution function of level set cannot converge and will spread alone with eyelashes, hence inaccurate result is obtained.

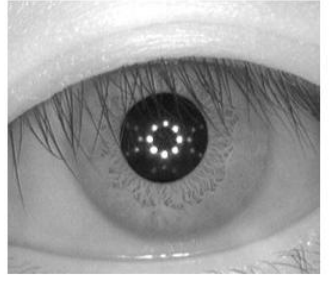

(a)

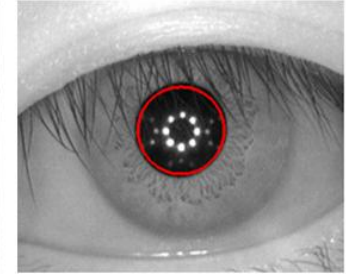

(b)

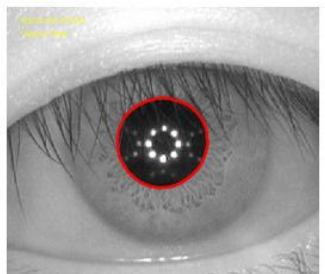

(c)

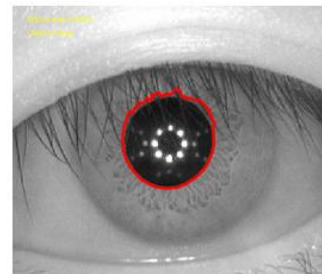

(d)

Figure 2. The process of fine pupil segmentaion. (a) Original iris image from CASIA-V3 Interval database, (b) Initial countour (result of coarse localization of pupil boundary), (c) Final countour after 20 iterations, (d) Final countour after 65 iterations. $\lambda_{1}=\lambda_{2}=1, \varepsilon=1, \mu=0.2 \cdot 255^{2}$. 
Here, we will discuss how to deal with this problem. As shown in Fig. 3, under the conditions that with the same initial level set, the same convergence threshold and different length parameter $\mu$, different pupil segmentation results are obtained. When $\mu=0.2 \cdot 255^{2}$, we get segmentation with burr, while when $\mu=5.0 \cdot 255^{2}$, almost perfect segmentation result is obtained.

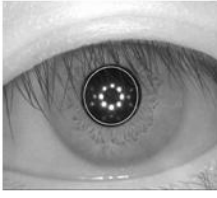

(a)

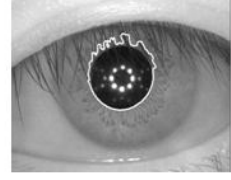

(b)

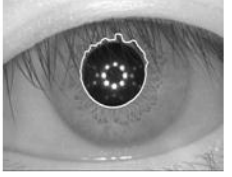

(c)

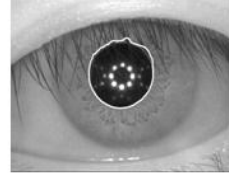

(d)

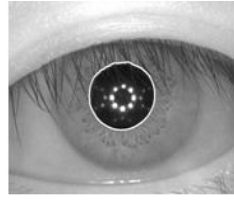

(e)

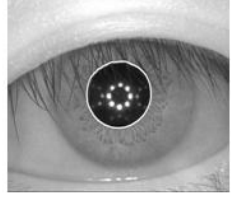

(f)

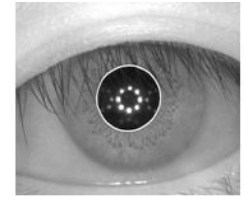

(g)

Figure 3. Pupil segmentation result with different parameter $\mu, \lambda_{1}=\lambda_{2}=1, \varepsilon=1, C T=2$. (a) Initial countour, (b) $\mu=0.2 \cdot 255^{2}$, (c) $\mu=1.0 \cdot 255^{2}$, (d) $\mu=2.0 \cdot 255^{2}$, (e) $\mu=3.0 \cdot 255^{2}$, (f) $\mu=4.0 \cdot 255^{2}$, (g) $\mu=5.0 \cdot 255^{2}$.

It is can be see that with the increase of $\mu$, we can get better segmentation result. Compare Fig. 3(b) with Fig. 3(e), we can find that parts of contour in Fig. 3(b) is not smooth and these parts have larger curvature, while Fig. 3(e) own relatively smoother contour, which is directly related to increased value of $\mu$. Nonetheless, just increasing value of $\mu$ can either increase the segmentation accuracy or increase actual iteration times. As shown in Fig. 4, under the conditions that with the same initial contour, the same convergence threshold and different length parameter $\mu$. We can see that set greater value of $\mu$ just lead to increase time complexity without any increase in segmentation accuracy. So how to set the parameter $\mu$ value will directly affect the pupil segmentation accuracy and the actual number of iteration.

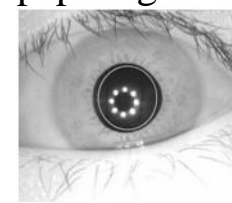

(a)

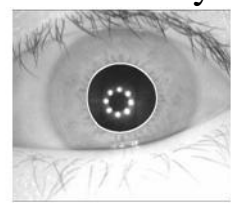

(b)

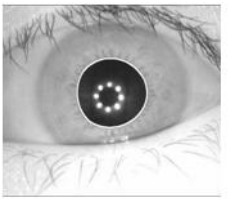

(c)

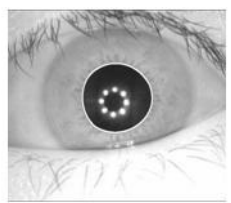

(d)

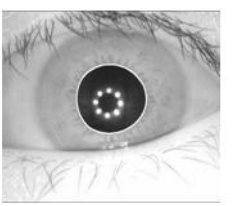

(e)

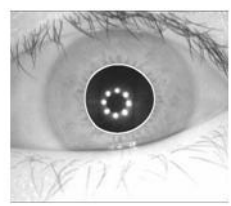

(f)

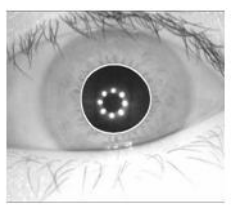

(g)

Figure 4. Pupil segmentation result with different parameter $\mu$ and different number of iterations, $\lambda_{1}=\lambda_{2}=1$, $\varepsilon=1, C T=2$. (a) Initial countour (b) $M O N T=16, \mu=0.2 \cdot 255^{2}$, (c) $M O N T=45, \mu=1.0255^{2}$, (d) $M O N T=62$, $\mu=2.0 \cdot 255^{2}$, (e) $M O N T=91, \mu=3.0 \cdot 255^{2}$, (f) $M O N T=125, \mu=4.0 \cdot 255^{2}$, (g) $M O N T=156, \mu=5.0 \cdot 255^{2}$.

From compare Fig. 3(a) with Fig. 4(a), it can be see that there is no eyelash in pupil area of Fig. 4(a), but the opposite is that there is eyelash interfere in pupil area of Fig. 3(a), in another word, we can think that Fig. 4(a) has better image quality than that of Fig. 3(a). Further, we can safely conclude that the related parameters of CV model should be self-adaptively set in accordance with iris image owns' features, and more importance is that the possible interference factors such as eyelashes, eyelids and specular reflections should be detected and evaluated to guide the related parameter setting to further expect evolution function of $\mathrm{CV}$ model can automated terminated

\section{Iris Segmentation}

As is known to all, if the eye are not open properly, certain regions of the iris will be highly occluded, which will further affect the segmentation process, and thus, the segmentation performance will be degraded. From Fig. 5 we can see that segmentation results are not remove eyelids interface.

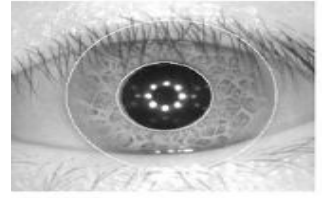

(a)

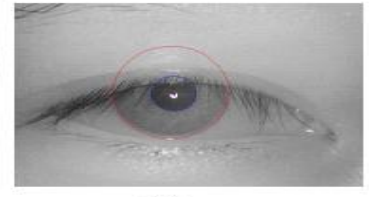

(b)

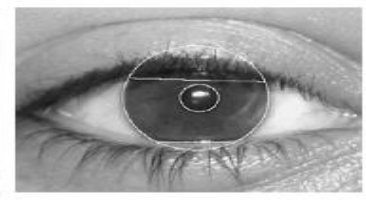

(c)

Fig. 5. Segmentation results from the published work. (a) Ghanizadeh et al. [12], (b) Zuo et al. [13], (c) Tan et al. [14].

CV model can deal with this situation, fromFig. 6 we can see that the interference factor of eyelids will be excluded out of the iris region. 


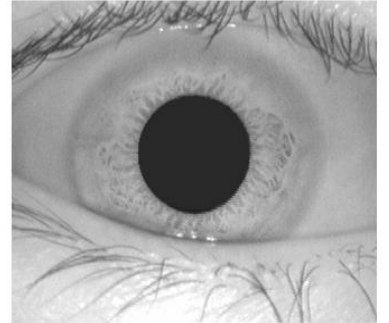

(a)

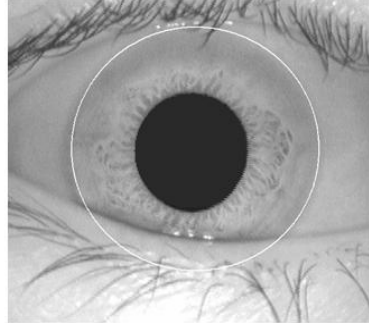

(b)

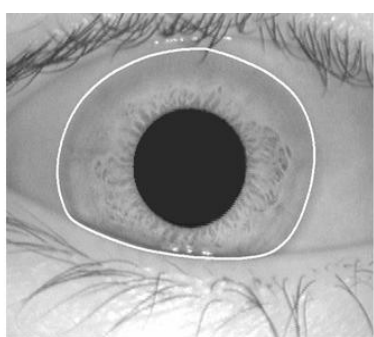

(c)

Figure 6. Iris segmentation result, $\mu=20 \cdot 255^{2}, \lambda_{1}=\lambda_{2}=1, \varepsilon=1, C T=2$. (a) Original iris image, (b) Initial countour, (c) Final countour,.

However, when eyelash interference appeared in iris area, CV model location accuracy maybe influenced.

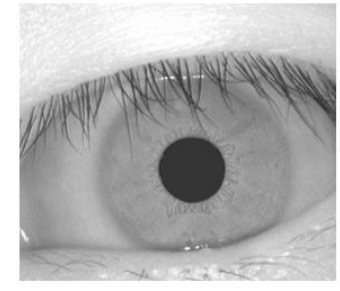

(a)

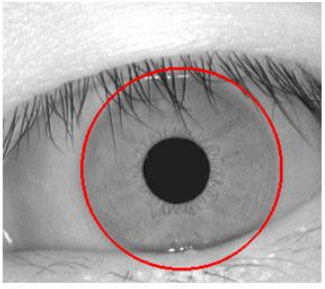

(b)

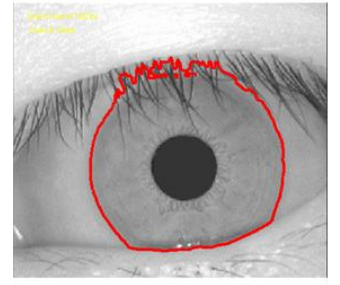

(c)

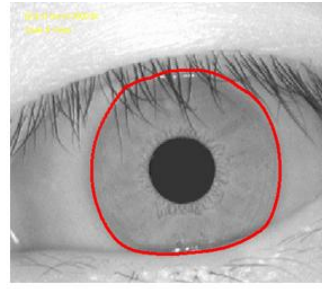

(d)

Figure 7. Iris segmentation result with different parameter $\mu, \lambda_{1}=\lambda_{2}=1, \varepsilon=1, C T=2$. (a) Original iris image, (b) Initial countour, (c) $M O N T=100, \mu=0.2 \cdot 255^{2}$, (d) $M O N T=2000, \mu=20 \cdot 255^{2}$.

As shown in Fig. 7(c), when $\mu$ is set as $0.2 .255^{2}, \mathrm{CV}$ model incorrectly considered that interfere eyelash is iris region, however, when parameter $\mu$ is set as $20.255^{2}$, CV model can get better segmentation result. Through the above-mentioned analysis, we can draw conclusion that to make CV model get fine segment pupil and iris area high accuracy, we just need to self-adaptively set the length parameter value according to iris images own unique features (MNOT and $C T$ keep as fixed value).

\section{Conclusion and Discussions}

Based on above circumstances, we can safely conclude that for different kinds of iris image, only to increase the value of $\mu$ cannot both improve pupil segmentation accuracy and reduce time complexity. The reason for above results is that when the value of $\mu$ is excessive big, which will not only influence the evolution speed of curve but also influence $\mu \cdot \operatorname{div}\left(\frac{\nabla \phi}{|\nabla \phi|}\right)$ to make $F\left(c_{1}, c_{2}, C\right)$ cannot get its smallest energy. Hence evolution function will iterate until reach the preset $M N O T$, this will inevitable increase time complexity. To solve this problem, two ways can be adopted. (1) Increase $C T$ value, (2) set a relatively smaller MNOT. However, these two strategies will bring new problem. Under the condition that with the same initial level set and the same length parameter $\mu$, when convergence condition has been set too loose or MNOT value has been set too small, both circumstances will lead to evolution function terminated earlier before it achieved the optimal segmentation result.

More attention will be paid to evaluating the proposed algorithm in more other iris image databases. In addition, we will continuously focus on analyze different interference factors that influence segmentation effect.

\section{Acknowledgements}

This research is supported by Jiangxi Province Education Department (Grant No. GJJ14542) and Nanchang Hangkong University (Grant No. EA201520009). 


\section{References}

[1] S. Shan, A. Ross. Iris segmentation using geodesic active contours. IEEE Transactions on Information Forensics and Security, 4 (2009), p. 824-36.

[2] J. Daugman. Statistical richness of visual phase information: Update on recognizing persons by iris patterns. Int J Comput Vision, 45 (2001), p. 25-38.

[3] T.N. Tan, Z.F. He, Z.Z. Sun. Efficient and robust segmentation of noisy iris images for non-cooperative iris recognition. Image Vision Comput, 28 (2010), p. 223-230.

[4] N.B. Puhan, N. Sunha, A.S. Kaushalram. Efficient segmentation technique for noisy frontal view iris images using Fourier spectral density. Signal Image Video P, 5 (2011), p. 105-119.

[5] K. Roy, P. Bhattacharya, C.Y. Suen. Iris segmentation using variational level set method. Optics and Lasers in Engineering, 49 (2011), p. 578-588.

[6] K. Roy, P. Bhattacharya, C.Y. Suen. Iris segmentation using game theory. Signal Image Video P, 6 (2012), p. 301-315.

[7] K. Roy, P. Bhattacharya, C.Y. Suen. Towards nonideal iris recognition based on level set method, genetic algorithms and adaptive asymmetrical SVMs. Engineering Applications of Artificial Intelligence, 24 (2011), p. 458-475.

[8] T.F. Chan, L.A. Vese. Active contours without edges. IEEE Transaction on Image processing, 10 (2001), p. 266-277.

[9] R. Malladi, J.J. Sethian, B.C. Vemuri. Shape Modeling with Front Propagaton: A Level Set Approach. IEEE Transaction on Pattern Analysis and Machine Intelligence, 17 (1995), p. 158-175.

[10] T. Dietenbeck, M. Alessandrini, D. Friboulet. CREASEG: a free software for the evaluation of image segmenation algorithm based on level-set. In IEEE International Conference On Image Processing, Hong Kong, China, 2010.

[11] CASIA Iris Image Databases. http:/www.cbsr.ia.ac.cn/IrisDatabase.htm.

[12] A. Ghanizadeh, A.A. Abarghouei, S. Sinaie, et al. Iris segmentation using an edge detector based on fuzzy sets theory and cellular learning automata. Applied Optics, 50 (2011) 3191-3200.

[13] J.Y. Zuo, N.A. Schmid. On a Methodology for Robust Segmentation of Nonideal Iris Images. IEEE Transactions on Systems Man and Cybernetics Bart B-Cybernetics, 40 (2010) 703-718.

[14] T.N. Tan, Z.F. He, Z.Z. Sun. Efficient and robust segmentation of noisy iris images for non-cooperative iris recognition. Image and Vision Computing, 28 (2010) 223-230. 